\title{
EQUIVALENT CHARACTERIZATIONS OF THE SPECTRA OF GRAPHS AND APPLICATIONS TO MEASURES OF DISTANCE-REGULARITY*
}

\author{
VICTOR DIEGO ${ }^{\dagger}$, JOSEP FÀBREGA ${ }^{\ddagger}$, AND MIQUEL ÀNGEL FIOL ${ }^{\S}$
}

\begin{abstract}
The spectrum of a graph usually provides a lot of information about its combinatorial structure. Moreover, from the spectrum, the so-called predistance polynomials can be defined, as a generalization, for any graph, of the distance polynomials of a distance-regular graph. Going further, the preintersection numbers generalize the intersection numbers of a distance-regular graph. This paper describes, for any graph, the closed relationships between its spectrum, predistance polynomials, and preintersection numbers. Then, some applications to derive combinatorial properties of the given graph, most of them related to some fundamental characterizations of distance-regularity, are presented. In particular, the so-called 'spectral excess theorem' is revisited. This result states that a connected regular graph is distance-regular if and only if its spectral excess, which is a value computed from the spectrum, equals the average excess, that is, the mean of the numbers of vertices at maximum distance from every vertex.
\end{abstract}

Key words. Graph, Spectrum, Predistance polynomials, Preintersection numbers, Distance-regular graph, Spectral excess theorem.

AMS subject classifications. 05E30, 05C50.

1. Preliminaries. Two main concepts involved in the study of a distance-regular graph $\Gamma$ are the intersection parameters and the distance polynomials. The former gives information about the combinatorial structure of $\Gamma$, whereas the latter constitutes an orthogonal sequence and yields the distance matrices of $\Gamma$. Moreover, both pieces of information are determined by the spectrum of $\Gamma$ (that is, by the eigenvalues and multiplicities of its adjacency matrix $\boldsymbol{A}$ ). Both concepts have been generalized for any graph (see Fiol and Garriga [19]) and, hence, they were called preintersection numbers and predistance polynomials. In this more general framework, it happens that some basic properties of the intersection numbers and the distance polynomials still hold. For instance, the preintersection numbers are somewhat related with the combinatorial properties of the graph, and the predistance polynomials are also orthogonal sequences having similar properties as the ones that inspired them.

In this paper, we first show that, for any graph $G$, the information contained in its spectrum, preintersection polynomials, and preintersection numbers is equivalent. To this end, we use both algebraic and combinatorial techniques. As a first application of our results, we derive some combinatorial properties of the given graphs, which can be inferred from any of the above three pieces of information. In particular, we present different characterization of different measures of distance-regularity.

In this context, recall that distance-regular graphs play a key role in the study of mathematical structures with a high degree of symmetry or regularity. Thus, since they were proposed by Biggs in the early

\footnotetext{
* Received by the editors on March 25, 2017. Accepted for publication on July 9, 2020. Handling Editor: Bryan L. Shader. Corresponding Author: Miquel Àngel Fiol. This research has been partially supported by AGAUR from the Catalan Government under project 2017SGR1087, and by MICINN from the Spanish Government under project PGC2018-095471-B-I00.

†(victordierrez@gmail.com).

${ }^{\ddagger}$ Departament de Matemàtiques, Universitat Politècnica de Catalunya, Barcelona, Catalonia, Spain (josep.fabrega@upc.edu).

$\S$ Departament de Matemàtiques, Universitat Politècnica de Catalunya, Barcelona, Catalonia, Spain, Barcelona Graduate School of Mathematics (miguel.angel.fiol@upc.edu).
} 
Electronic Journal of Linear Algebra, ISSN 1081-3810

70's, the distance-regular graphs have been the subject of an intensive study, which includes numerous characterizations, both combinatorial and algebraic. As an example of the former, Rowlinson [24] showed that a graph is distance-regular if and only if the number of walks of a given length between two vertices depends only on the distance between such vertices.

A quasi-spectral characterization of distance-regular graphs, which is due to Fiol and Garriga [19], is known in the literature as the 'spectral excess theorem'. This result states that a graph $G$ with $d+1$ distinct eigenvalues is distance-regular if and only if its spectral excess (a number computed from the adjacency spectrum of $G$ ) equals its mean excess (that is, the mean number of vertices at maximum distance $d$ from each vertex). Apart from the spectrum, the predistance polynomials are a key concept in the proof of the spectral excess theorem. Since its inception, this theorem has given rise to several variants, concerning both specific families of distance-regular graphs and other more general combinatorial structures. For instance, these include the completely regular codes [6], and $P$-polynomial association schemes ([22], where the predistance polynomials are also used in the proofs. Moreover, the spectral excess theorem has been a key tool for proving other important results, such as the construction, by Van Dam and Koolen [14], of the first infinite family of distance-regular graphs that are not vertex-transitive.

Thus, the main purpose of this paper is to show how our three pieces of information, spectrum, predistance polynomials and preintersection numbers, can be used both to give alternative characterizations of some of the above distance-regularity properties, and to derive some new related results.

For more background on spectra of graphs, distance-regular graphs, and their characterizations, we refer to the reader to $[2,3,5,7,15,17,20]$.

2. Three equivalent pieces of information. In this section, we define our three pieces of information and their basic properties. First, let us recall some basic concepts, notation, and results on which our study is based. Throughout the paper, $\Gamma=(V, E)$ stands for a (simple and finite) connected graph with vertex set $V$ and edge set $E$. We denote by $n$ the number of vertices and by $e$ the number of edges. Adjacency between vertices $u$ and $v(u v \in E)$ will be denoted by $u \sim v$. The adjacency matrix $\boldsymbol{A}$ of $\Gamma$ is the $(0,1)$-matrix with rows and columns indexed by the vertices, such that $(\boldsymbol{A})_{u v}=1$ if and only if $u \sim v$.

2.1. The spectrum. One of the most important tools in the study of the algebraic properties of a graph $\Gamma$ is its spectrum. The spectrum of $\Gamma$ is the set of eigenvalues of its adjacency matrix $\boldsymbol{A}$ together with their multiplicities:

$$
\operatorname{sp} \Gamma=\left\{\lambda_{0}^{m_{0}}, \lambda_{1}^{m_{1}}, \ldots, \lambda_{d}^{m_{d}}\right\},
$$

where $\lambda_{0}>\lambda_{1}>\cdots>\lambda_{d}$ and, for $i=0, \ldots, d$, the superscript $m_{i}=m\left(\lambda_{i}\right)$ stand for the multiplicity of the eigenvalue $\lambda_{i}$. Notice that, since $\Gamma$ is connected, $m_{0}=1$, and if $\Gamma$ is $k$-regular, then $\lambda_{0}=k$. Throughout the paper, $d$ will always denote the number of distinct eigenvalues minus one.

2.2. The predistance polynomials. Given a graph $\Gamma$ with spectrum as above, the predistance polynomials $p_{0}, \ldots, p_{d}$, introduced by Fiol and Garriga in [19], are polynomials in $\mathbb{R}_{d}[x]$ (that is, the vector space of real polynomials with degree at most $d$ ), with degree $\operatorname{deg}\left(p_{i}\right)=i$, which are orthogonal with respect to the scalar product

$$
\langle f, g\rangle_{\Gamma}=\frac{1}{n} \operatorname{tr}(f(\boldsymbol{A}) g(\boldsymbol{A}))=\frac{1}{n} \sum_{i=0}^{d} m_{i} f\left(\lambda_{i}\right) g\left(\lambda_{i}\right),
$$


Electronic Journal of Linear Algebra, ISSN 1081-3810

A publication of the International Linear Algebra Society

Volume 36, pp. 629-644, September 2020.

631

Equivalent Characterizations of the Spectra of Graphs and Applications

and normalized in such a way that $\left\|p_{i}\right\|_{\Gamma}^{2}=p_{i}\left(\lambda_{0}\right)$ (this always makes sense since it is known that $p_{i}\left(\lambda_{0}\right)>0$ for every $i=0, \ldots, d)$. (Notice that such polynomials are determined by the spectrum, for instance, through the Gram-Schmidt method; see Subsection 3.1.)

Some basic properties of these polynomials, which can be seen as a generalization of the distance polynomials of a distance-regular graph, are given in the following lemma, see [6].

LEMMA 2.1. Let $\Gamma$ be a graph with average degree $\bar{k}=2 e / n$, predistance polynomials $p_{i}$, and consider their sums $q_{i}=p_{0}+\cdots+p_{i}$, for $i=0, \ldots, d$. Then,

(a) $p_{0}=1, p_{1}=\left(\lambda_{0} / \bar{k}\right) x$, and the constants of the three-term recurrence

$$
x p_{i}=\beta_{i-1} p_{i-1}+\alpha_{i} p_{i}+\gamma_{i+1} p_{i+1},
$$

where $\beta_{-1}=\gamma_{d+1}=0$, satisfy:

(a1) $\alpha_{i}+\beta_{i}+\gamma_{i}=\lambda_{0}$, for $i=0, \ldots, d$;

(a2) $p_{i-1}\left(\lambda_{0}\right) \beta_{i-1}=p_{i}\left(\lambda_{0}\right) \gamma_{i}$, for $i=1, \ldots, d$.

(b) $p_{d}\left(\lambda_{0}\right)=n\left(\sum_{i=0}^{d} \frac{\pi_{0}^{2}}{m_{i} \pi_{i}^{2}}\right)^{-1}$, where $\pi_{i}=\prod_{j \neq i}\left|\lambda_{i}-\lambda_{j}\right|$, for $i=0, \ldots, d$.

(c) $1=q_{0}\left(\lambda_{0}\right)<q_{1}\left(\lambda_{0}\right)<\cdots<q_{d}\left(\lambda_{0}\right)=n$, and $q_{d}\left(\lambda_{i}\right)=0$ for every $i \neq 0$. Thus, $q_{d}=H$ is the Hoffman polynomial characterizing the regularity of $\Gamma$ by the condition $H(\boldsymbol{A})=\boldsymbol{J}$, where $\boldsymbol{J}$ stands for the all ones matrix (see Hoffman [21]).

(d) The three-term recurrence $(2.3)$ can be represented through a tridiagonal $(d+1) \times(d+1)$ matrix $\boldsymbol{R}$ such that, in the quotient ring $\mathbb{R}[x] /(m)$, where $(m)$ is the ideal generated by the minimal polynomial $m=\prod_{i=0}^{d}\left(x-\lambda_{i}\right)$ of $\boldsymbol{A}$, it satisfies

$$
x \boldsymbol{p}=x\left(\begin{array}{c}
p_{0} \\
p_{1} \\
p_{2} \\
\vdots \\
p_{d}
\end{array}\right)=\left(\begin{array}{ccccc}
\alpha_{0} & \gamma_{1} & & & \\
\beta_{0} & \alpha_{1} & \gamma_{2} & & \\
& \beta_{1} & \alpha_{2} & & \\
& & & \ddots & \gamma_{d} \\
& & & \beta_{d-1} & \alpha_{d}
\end{array}\right)\left(\begin{array}{c}
p_{0} \\
p_{1} \\
p_{2} \\
\vdots \\
p_{d}
\end{array}\right)=\boldsymbol{R} \boldsymbol{p}
$$

2.3. The preintersection numbers. The preintersection numbers can be seen as a generalization of the intersection numbers of a distance-regular graph, which are closely related to its combinatorial properties (see e.g. Biggs [2]). In the more general context of any graph, the preintersection numbers give us an algebraic information on the graph, which is of the same nature as the spectrum of its adjacency matrix. More precisely, the preintersection numbers $\xi_{i j}^{h}, i, j, h \in\{0, \ldots, d\}$, are the Fourier coefficients of $p_{i} p_{j}$ in terms of the basis $\left\{p_{h}\right\}_{0 \leq h \leq d}$, that is,

$$
\xi_{i j}^{h}=\frac{\left\langle p_{i} p_{j}, p_{h}\right\rangle_{\Gamma}}{\left\|p_{h}\right\|_{\Gamma}^{2}}=\frac{1}{n p_{h}\left(\lambda_{0}\right)} \sum_{r=0}^{d} m\left(\lambda_{r}\right) p_{i}\left(\lambda_{r}\right) p_{j}\left(\lambda_{r}\right) p_{h}\left(\lambda_{r}\right) .
$$

Notice that, in particular, the coefficients of the three-term recurrence $(2.3)$ are $\alpha_{i}=\xi_{1, i}^{i}, \beta_{i}=\xi_{1, i+1}^{i}$, and $\gamma_{i}=\xi_{1, i-1}^{i}$. In fact, from our derivations it will be clear that such coefficients determine all the other preintersection numbers.

3. Formulas and procedures for equivalence. In this section, we study the equivalence between the three pieces of information described in Section 2. Namely, the spectrum, the predistance polynomials, and the preintersection numbers of a given graph. 
3.1. From the spectrum to the predistance polynomials. As mentioned above, the spectrum of a graph plays a central role in the study of its algebraic and combinatorial properties. To obtain the predistance polynomials introduced in Subsection 2.2 we consider the scalar product defined in (2.2) and apply the Gram-Schmidt orthogonalization method to the basis $\left\{1, x, \ldots, x^{d}\right\}$, normalizing the obtained sequence of orthogonal polynomials in such a way that $\left\|p_{i}\right\|^{2}=p_{i}\left(\lambda_{0}\right)$. (This makes sense since, from the theory of orthogonal polynomials, it is known that $p_{i}\left(\lambda_{0}\right)>0$ for any $i=0, \ldots, d$.) As mentioned in Lemma 2.1, $H=p_{0}+\cdots+p_{d}$ is the Hoffman polynomial satisfying $H\left(\lambda_{i}\right)=0$ for $i>0, H\left(\lambda_{0}\right)=n$, and characterizing the regularity of the graph by the condition $H(\boldsymbol{A})=\boldsymbol{J}$.

3.2. From the predistance polynomials to the spectrum. In this subsection, we show how the spectrum of a graph $\Gamma$ can be obtained from its predistance polynomials.

Proposition 3.1. Let $p_{0}, p_{1}, \ldots, p_{d}$ be the predistance polynomials of a graph $\Gamma$, with $\omega_{i}^{j}$ being the coefficient of $x^{j}$ in $p_{i}$. Then,

(a) The different eigenvalues $\lambda_{i} \neq \lambda_{0}$ of $\Gamma$ are the $d$ distinct zeros of the Hoffman polynomial $H=$ $p_{0}+p_{1}+\cdots+p_{d}$.

(b) The largest eigenvalue (spectral radius) is

$$
\lambda_{0}=-\frac{\omega_{1}^{1} \omega_{2}^{0}}{\omega_{2}^{2}}
$$

(c) The multiplicity of the eigenvalue $\lambda_{i}$, for $i=0, \ldots, d$, is

$$
m_{i}=n\left(\sum_{j=0}^{d} \frac{p_{j}\left(\lambda_{i}\right)^{2}}{p_{j}\left(\lambda_{0}\right)}\right)^{-1} .
$$

Proof. (a) As mentioned in Lemma 2.1, $H=p_{0}+\cdots+p_{d}$ is the Hoffman polynomial satisfying $H\left(\lambda_{i}\right)=0$ for $i=1, \ldots, d$.

(b) The expressions for $p_{0}$ and $p_{1}$ (see Lemma $2.1(a)$ ) imply that $\omega_{0}^{0}=1$ and $\omega_{1}^{0}=0$. Then,

$$
\alpha_{0}=0, \quad \alpha_{1}=-\frac{\omega_{2}^{1}}{\omega_{2}^{2}}, \quad \text { and } \quad \beta_{0}=-\frac{\omega_{1}^{1}}{\omega_{2}^{2}} \omega_{2}^{0},
$$

and (3.6) follows from $\lambda_{0}=\alpha_{0}+\beta_{0}$.

(c) See the proof of Proposition 3.6 (b). This is a result from [17]. (The corresponding result for distanceregular graphs was first proved by Biggs in [2].)

The spectral radius can also be determined as the largest root of the polynomial

$$
h=\left(\sum_{i=1}^{d} \frac{\lambda_{i}}{p_{d}\left(\lambda_{i}\right)} \prod_{\substack{j=1 \\ j \neq i}}^{d} \frac{x-\lambda_{j}}{\lambda_{i}-\lambda_{j}}\right) p_{d}(x)-x .
$$

This comes from the combination of the following two facts: the multiplicity of each eigenvalue can be also calculated as

$$
m_{i}=\frac{\phi_{0} p_{d}\left(\lambda_{0}\right)}{\phi_{i} p_{d}\left(\lambda_{i}\right)}, \quad \text { for } i=0, \ldots, d,
$$


where $\phi_{i}=\prod_{j=0, j \neq i}^{d}\left(\lambda_{0}-\lambda_{j}\right)$, see [17], and the sum of all the eigenvalues has to be zero, $\sum_{i=0}^{d} m_{i} \lambda_{i}=$ $\operatorname{tr} \boldsymbol{A}=0$. Note that, in fact, the polynomial $h$ has also the roots $\lambda_{1}, \ldots, \lambda_{d}$.

Another approach is to notice that each coefficient of $H(x)=\sum_{j=0}^{d} h_{j} x^{j}$ can be written as $h_{j}=$ $w_{j}^{j}+w_{j+1}^{j}+\cdots+w_{d}^{j}$, where $\omega_{i}^{j}$ is the coefficient of degree $j$ of the polynomial $p_{i}$. In particular, if $\Gamma$ is regular, then

$$
H(x)=\frac{n}{\pi_{0}} \prod_{i=1}^{d}\left(x-\lambda_{i}\right)=\frac{n}{\pi_{0}} \sum_{C \subset[d]}(-1)^{|C|} x^{d-|C|}\left(\prod_{j \in C} \lambda_{j}\right),
$$

where $[d]=\{0,1, \ldots, d\}, \pi_{0}=\prod_{j=1}^{d}\left|\lambda_{0}-\lambda_{j}\right|$, and, hence, we have the system of $d$ equations

$$
h_{j}=w_{j}^{j}+w_{j+1}^{j}+\cdots+w_{d}^{j}=\frac{n}{\pi_{0}} \sum_{|C|=d-j}(-1)^{d-j}\left(\prod_{i \in C} \lambda_{i}\right), \quad j=0, \ldots, d-1,
$$

with unknowns $\lambda_{1}, \ldots, \lambda_{d}$.

3.3. From the predistance polynomials to the preintersection numbers. In this subsection, we assume that the predistance polynomials of a graph $\Gamma$ are given and, from them, we want to obtain its preintersection numbers. Of course, we could do so by applying (2.5), but this requires to know the spectrum of $\Gamma$, which requires an intermediate computation (as shown in Subsection 3.2). Consequently, we want to directly relate the preintersection numbers to (the coefficients of) the predistance polynomials. With this aim, we use both the three-term recurrence (2.3) and the generic expression of each polynomial $p_{i}$ as above. This leads to the following result.

Proposition 3.2. Given the predistance polynomials of a graph $\Gamma, p_{i}=\sum_{j=0}^{i} \omega_{i}^{j} x^{j}$, its preintersection numbers are:

(a) $\alpha_{0}=-\frac{\omega_{1}^{0}}{\omega_{1}^{1}}, \quad \alpha_{i}=\frac{\omega_{i}^{i-1}}{\omega_{i}^{i}}-\frac{\omega_{i+1}^{i}}{\omega_{i+1}^{i+1}} \quad(1 \leq i \leq d-1) ;$

(b) $\beta_{i}=\frac{\omega_{i+1}^{i-1}}{\omega_{i}^{i}}-\frac{\omega_{i+1}^{i}}{\omega_{i}^{i}}\left(\frac{\omega_{i+1}^{i}}{\omega_{i+1}^{i+1}}-\frac{\omega_{i+2}^{i+1}}{\omega_{i+2}^{i+2}}\right)-\frac{\omega_{i+1}^{i+1}}{\omega_{i+2}^{i+2}} \frac{\omega_{i+2}^{i}}{\omega_{i}^{i}} \quad(0 \leq i \leq d-2)$;

(c) $\gamma_{i}=\frac{\omega_{i-1}^{i-1}}{\omega_{i}^{i}} \quad(1 \leq i \leq d)$.

Proof. By using the expressions of $p_{i-1}, p_{i}$, and $p_{i+1}$ in (2.3), and considering the terms of degree $i+1$, we get

$$
\omega_{i}^{i}=\gamma_{i+1} \omega_{i+1}^{i+1}, \quad i=0, \ldots, d-1
$$

giving $(c)$.

Analogously, from the term of degree $i$, we have

$$
\omega_{i}^{i-1}=\alpha_{i} \omega_{i}^{i}+\gamma_{i+1} \omega_{i+1}^{i}
$$

whence, by using the value of $\gamma_{i+1}$, we obtain

$$
\omega_{i}^{i-1}=\alpha_{i} \omega_{i}^{i}+\frac{\omega_{i}^{i}}{\omega_{i+1}^{i+1}} \omega_{i+1}^{i}
$$

giving $(a)$ for $1 \leq i \leq d-1$. The value of $\alpha_{0}$ is obtained from (2.3) with $i=0$ and the value of $\gamma_{1}$. 
Finally, looking at the terms of degree $i-1$ :

$$
\omega_{i}^{i-2}=\beta_{i-1} \omega_{i-1}^{i-1}+\alpha_{i} \omega_{i}^{i-1}+\gamma_{i+1} \omega_{i+1}^{i-1},
$$

and using the values for $\alpha_{i}$ and $\gamma_{i+1}$, we have

$$
\omega_{i}^{i-2}=\beta_{i-1} \omega_{i-1}^{i-1}+\left(\frac{\omega_{i}^{i-1}}{\omega_{i}^{i}}-\frac{\omega_{i+1}^{i}}{\omega_{i+1}^{i+1}}\right) \omega_{i}^{i-1}+\frac{\omega_{i-1}^{i-1}}{\omega_{i}^{i}} \omega_{i+1}^{i-1} .
$$

This yields the value of $\beta_{i}$ for $1 \leq i \leq d-2$. The value of $\beta_{0}$ is obtained from (2.3) with $i=1$, and the values of $\alpha_{1}$ and $\gamma_{2}$. This also yields (b) with $i=0$, by setting $\omega_{1}^{-1}=0$.

Note that, in the above result, $\alpha_{d}$ and $\beta_{d-1}$ do not need to be mentioned, since they are computed by using Lemma $2.1(a 1)$ with $\lambda_{0}=\alpha_{0}+\beta_{0}$.

A matrix approach. The above computation can be also carried out by using a matrix approach. To this end, let us consider the given matrices

$$
\boldsymbol{\Omega}=\left(\begin{array}{ccccc}
\omega_{0}^{0} & & & & \\
\omega_{1}^{0} & \omega_{1}^{1} & & & \\
\omega_{2}^{0} & \omega_{2}^{0} & \omega_{2}^{2} & & \\
\vdots & & & \ddots & \\
\omega_{d}^{0} & & \ldots & & \omega_{d}^{d}
\end{array}\right) \text { and } \boldsymbol{U}=\left(\begin{array}{ccccc}
0 & 1 & 0 & \cdots & 0 \\
0 & 0 & 1 & & \\
\vdots & & & \ddots & \\
0 & \cdots & & 0 & 1 \\
0 & 0 & 0 & \cdots & 0
\end{array}\right)
$$

where, as above, the $\omega_{i}^{j}, i, j=0, \ldots, d$ stand for the coefficients of the predistance polynomials, and $\boldsymbol{U}$ is a $(d+1) \times(d+1)$ matrix. From them, we want to find the tridiagonal matrix of the preintersection numbers of $\Gamma$ :

$$
\boldsymbol{R}=\left(\begin{array}{ccccc}
\alpha_{0} & \gamma_{1} & & & \\
\beta_{0} & \alpha_{1} & \gamma_{2} & & \\
& \beta_{1} & \alpha_{2} & & \\
& & & \ddots & \gamma_{d} \\
& & & \beta_{d-1} & \alpha_{d}
\end{array}\right)
$$

Then, we have the following result.

Proposition 3.3. Let $\Gamma$ be a graph with predistance polynomials $p_{0}, \ldots, p_{d}$, and coefficient matrix $\boldsymbol{\Omega}$. Let $\boldsymbol{\Omega}^{\prime}$ and $\boldsymbol{R}^{\prime}$ be the matrices obtained, respectively, from $\boldsymbol{\Omega}$ and $\boldsymbol{R}$ by removing its last row. Then,

$$
\boldsymbol{R}^{\prime}=\boldsymbol{\Omega}^{\prime} \boldsymbol{U} \boldsymbol{\Omega}^{-1},
$$

Proof. By using the (column) vectors $\boldsymbol{p}=\left(p_{0}, p_{1}, \ldots, p_{d}\right)^{\top}$ and $\boldsymbol{x}=\left(1, x, \ldots, x^{d}\right)^{\top}$, and $\boldsymbol{p}^{\prime}$ and $\boldsymbol{x}^{\prime}$ obtained from $\boldsymbol{p}$ and $\boldsymbol{x}$ by deleting the last entry, we have $\boldsymbol{p}=\boldsymbol{\Omega} \boldsymbol{x}, \boldsymbol{p}^{\prime}=\boldsymbol{\Omega}^{\prime} \boldsymbol{x}^{\prime}$, and $x \boldsymbol{x}^{\prime}=\boldsymbol{U} \boldsymbol{x}$. Moreover, the first $d$ equations in (2.4) are $x \boldsymbol{p}^{\prime}=\boldsymbol{R}^{\prime} \boldsymbol{p}$. Then, all together yields

$$
x \boldsymbol{\Omega}^{\prime} \boldsymbol{x}^{\prime}=\boldsymbol{\Omega}^{\prime} \boldsymbol{U} \boldsymbol{x}=\boldsymbol{R}^{\prime} \boldsymbol{\Omega} \boldsymbol{x},
$$

so that $\left(\boldsymbol{\Omega}^{\prime} \boldsymbol{U}-\boldsymbol{R}^{\prime} \boldsymbol{\Omega}\right) \boldsymbol{x}=\mathbf{0}$ and, then, it must be $\boldsymbol{\Omega}^{\prime} \boldsymbol{U}=\boldsymbol{R}^{\prime} \boldsymbol{\Omega}$, whence the result follows.

Finally, the last row of $\boldsymbol{R}$ is computed by using Lemma $2.1(a 1)$. 
3.4. From the preintersection numbers to the predistance polynomials. To obtain the predistance polynomials from the preintersection numbers of a graph $\Gamma$, we just need to apply the three-term recurrence (2.3) which, initialized by $p_{0}=1$, yields:

$$
p_{i}=\frac{1}{\gamma_{i}}\left[\left(x-\alpha_{i-1}\right) p_{i-1}-\beta_{i-2} p_{i-2}\right], \quad i=1, \ldots, d
$$

In particular, as stated in Lemma 2.1 $(a)$, we get $p_{1}=\left(\lambda_{0} / \bar{k}\right) x$, so that $\Gamma$ is regular if and only if $p_{1}=x$.

Alternatively, we can also compute $p_{i}$ directly by using the principal submatrix of the recurrence matrix $\boldsymbol{R}$ in (2.4). Namely,

$$
\boldsymbol{R}_{i}=\left(\begin{array}{ccccc}
\alpha_{0} & \gamma_{1} & & & \\
\beta_{0} & \alpha_{1} & \gamma_{2} & & \\
& \beta_{1} & \alpha_{2} & & \\
& & & \ddots & \gamma_{i} \\
& & & \beta_{i-1} & \alpha_{i}
\end{array}\right), \quad i=0,1, \ldots, d
$$

Proposition 3.4. The predistance polynomial $p_{i}$ associated to the recurrence matrix $\boldsymbol{R}$ is

$$
p_{i}=\frac{1}{\gamma_{0} \cdots \gamma_{i}} p_{c}\left(\boldsymbol{R}_{i-1}\right), \quad i=1, \ldots, d,
$$

where $p_{c}\left(\boldsymbol{R}_{i-1}\right)$ stands for the characteristic polynomial of $\boldsymbol{R}_{i-1}$.

Proof. We use induction on the degree. The result holds for $i=1,2$ since, by (3.12), we get

$$
p_{1}=\frac{1}{\gamma_{1}}\left(x-\alpha_{0}\right)=\frac{1}{\gamma_{1}} \boldsymbol{p}_{c}\left(\boldsymbol{R}_{0}\right) \quad \text { and } \quad p_{2}=\frac{1}{\gamma_{1} \gamma_{2}}\left[\left(x-\alpha_{0}\right)\left(x-\alpha_{1}\right)-\beta_{0} \gamma_{1}\right]=\frac{1}{\gamma_{1} \gamma_{2}} p_{c}\left(\boldsymbol{R}_{1}\right) \text {. }
$$

Then, we assume that the result holds for all values smaller than $i(\geq 3)$ and prove that $\operatorname{det}\left(x \boldsymbol{I}-\boldsymbol{R}_{i-1}\right)=p_{i}$ developing by expansion of the determinant along its last column.

Also, we can obtain explicit formulas for the coefficients of the polynomials in terms of the preintersection numbers.

LEMMA 3.5. Given the preintersection numbers $\alpha_{i}, \beta_{i}$, and $\gamma_{i}$ of a graph $\Gamma$, the three coefficients of the higher degree terms of its predistance polynomial $p_{i}=\omega_{i}^{i} x^{i}+\omega_{i}^{i-1} x^{i-1}+\cdots+\omega_{i}^{0}$, are:

(i) $\omega_{i}^{i}=\frac{1}{\gamma_{1} \gamma_{2} \cdots \gamma_{i}}$

(ii) $\omega_{i}^{i-1}=-\frac{\alpha_{0}+\cdots+\alpha_{i-1}}{\gamma_{1} \gamma_{2} \cdots \gamma_{i}}$;

(iii) $\omega_{i}^{i-2}=\frac{\sum_{0 \leq r<s \leq i-1} \alpha_{r} \alpha_{s}-\sum_{r=0}^{i-2} \beta_{r} \gamma_{r+1}}{\gamma_{1} \gamma_{2} \cdots \gamma_{i}}$.

Proof. By using induction with the three-term recurrence (2.3), we get:

( $i)$ The principal coefficient of the polynomial $p_{i}=\frac{1}{\gamma_{i}}\left[\left(x-\alpha_{i-1}\right) p_{i-1}-\beta_{i-2} p_{i-2}\right]$ is the principal coefficient of $\frac{1}{\gamma_{i}} p_{i-1}$, that is, $\frac{1}{\gamma_{i}} \omega_{i-1}^{i-1}$.

(ii) The second coefficient of $p_{i}$ can be expressed in terms of the previous coefficients as:

$$
\omega_{i}^{i-1}=\frac{\omega_{i-1}^{i-2}-\alpha_{i-1} \omega_{i-1}^{i-1}}{\gamma_{i}}
$$


and using the first statement we have:

$$
\omega_{i}^{i-1}=\frac{\omega_{i-1}^{i-2}}{\gamma_{i}}-\alpha_{i-1} \frac{1}{\gamma_{2} \cdots \gamma_{i}} .
$$

(iii) For the coefficient of the third highest degree term, we get:

$$
\omega_{i}^{i-2}=\frac{\omega_{i-1}^{i-3}-\alpha_{i-1} \omega_{i-1}^{i-2}-\beta_{i-2} \omega_{i-2}^{i-2}}{\gamma_{i}},
$$

which, in addition with the previous results, it can be expressed as:

$$
\omega_{i}^{i-2}=\frac{\omega_{i-1}^{i-3}}{\gamma_{i}}-\frac{\alpha_{1} \alpha_{i-1}+\cdots+\alpha_{i-2} \alpha_{i-1}}{\gamma_{2} \cdots \gamma_{i}}-\frac{\beta_{i-2} \gamma_{i-1}}{\gamma_{2} \cdots \gamma_{i}} .
$$

Of course, this procedure can be carried on by calculating each $\omega_{i}^{j}$ from the three-term recurrence and using the expressions of the previously computed $\omega_{i}^{i}, \ldots, \omega_{i}^{j+1}$.

The above computations can also be performed by using a matrix approach. Indeed, they can be set as a linear system by using the matrix approach in Proposition 3.3 of the previous subsection.

3.5. From the preintersection numbers to the spectrum. Let us now see how the preintersection numbers of a graph allow us to compute its spectrum.

Proposition 3.6. Given a graph $\Gamma$ with $d+1$ distinct eigenvalues and matrix $\boldsymbol{R}$ of preintersection numbers, its spectrum $\operatorname{sp} \Gamma=\left\{\lambda_{0}^{m_{0}}, \lambda_{1}^{m_{1}}, \ldots, \lambda_{d}^{m_{d}}\right\}$ can be computed in the following way:

(a) The different eigenvalues $\lambda_{0}>\lambda_{1}>\cdots>\lambda_{d}$ of $\Gamma$ are the eigenvalues of $\boldsymbol{R}$, that is, the (distinct) zeros of its characteristic polynomial $p_{c}(\boldsymbol{R})=\operatorname{det}(x \boldsymbol{I}-\boldsymbol{R})$.

(b) Let $\boldsymbol{u}_{i}$ and $\boldsymbol{v}_{i}$ be the 'standard' (that is, normalized with first component 1 ) left and right eigenvectors corresponding to $\lambda_{i}$. Then, the multiplicities are given by the formulas

$$
m_{i}=\frac{n}{\left\langle\boldsymbol{u}_{i}, \boldsymbol{v}_{i}\right\rangle}, \quad i=0, \ldots, d,
$$

where $n=\left\langle\boldsymbol{u}_{0}, \boldsymbol{v}_{0}\right\rangle$ is the number of vertices of $\Gamma$.

Proof. Let $\boldsymbol{P}$ be the matrix indexed with $0, \ldots, d$, and with entries $\boldsymbol{P}_{i j}=p_{i}\left(\lambda_{j}\right)$. Then, because of (2.4), its $i$-th column $\boldsymbol{v}_{i}$ is a right $\lambda_{i}$-eigenvector of the recurrence matrix $\boldsymbol{R}: \boldsymbol{R P}=\boldsymbol{P} \boldsymbol{D}$, where $\boldsymbol{D}=$ $\operatorname{diag}\left(\lambda_{0}, \ldots, \lambda_{d}\right)$. Then, as $\boldsymbol{P}^{-1} \boldsymbol{R}=\boldsymbol{D} \boldsymbol{P}^{-1}$, the $i$-th row $\boldsymbol{u}_{i}$ of $\boldsymbol{P}^{-1}$ is a left $\lambda_{i}$-eigenvector of $\boldsymbol{R}$. Moreover, because of the orthogonal property of the predistance polynomials with respect to the scalar product (2.2), the inverse of the matrix $\boldsymbol{P}$ is

$$
\boldsymbol{P}^{-1}=\frac{1}{n}\left(\begin{array}{cccc}
m_{0} \frac{p_{0}\left(\lambda_{0}\right)}{n_{0}} & m_{0} \frac{p_{1}\left(\lambda_{0}\right)}{n_{1}} & \cdots & m_{0} \frac{p_{d}\left(\lambda_{0}\right)}{n_{d}} \\
m_{1} \frac{p_{0}\left(\lambda_{1}\right)}{n_{0}} & m_{1} \frac{p_{1}\left(\lambda_{1}\right)}{n_{1}} & \cdots & m_{1} \frac{p_{d}\left(\lambda_{1}\right)}{n_{d}} \\
\vdots & \vdots & & \vdots \\
m_{d} \frac{p_{0}\left(\lambda_{d}\right)}{n_{0}} & m_{d} \frac{p_{1}\left(\lambda_{d}\right)}{n_{1}} & \cdots & m_{d} \frac{p_{d}\left(\lambda_{d}\right)}{n_{d}}
\end{array}\right),
$$

where $n_{i}=p_{i}\left(\lambda_{0}\right)$. Then, from $\left(\boldsymbol{P}^{-1} \boldsymbol{P}\right)_{i i}=1,0 \leq i \leq d$, we get

$$
m_{i}=n\left(\sum_{j=0}^{d} \frac{p_{j}\left(\lambda_{i}\right)^{2}}{p_{j}\left(\lambda_{0}\right)}\right)^{-1}=\frac{n}{\left\langle\boldsymbol{u}_{i}, \boldsymbol{v}_{i}\right\rangle}, \quad i=0, \ldots, d,
$$

as claimed. Finally, notice that, as $m_{0}=1, n=\left\langle\boldsymbol{u}_{0}, \boldsymbol{v}_{0}\right\rangle$. 
Note also that, in (3.15), the right and left eigenvectors are, respectively, $\boldsymbol{v}_{i}=\left(p_{0}\left(\lambda_{i}\right), p_{1}\left(\lambda_{i}\right), \ldots, p_{d}\left(\lambda_{i}\right)\right)^{\top}$, and $\boldsymbol{u}_{i}=\left(\frac{p_{0}\left(\lambda_{i}\right)}{p_{0}\left(\lambda_{0}\right)}, \frac{p_{1}\left(\lambda_{i}\right)}{p_{1}\left(\lambda_{0}\right)}, \ldots, \frac{p_{d}\left(\lambda_{i}\right)}{p_{d}\left(\lambda_{0}\right)}\right)$. In the particular case when $\Gamma$ is a distance-regular graph, an alternative proof of (3.14) without using the orthogonal polynomials was given by Biggs [2].

3.6. From the spectrum to the preintersection numbers. As far as we know, in the case of distance-regular graphs there are no formulas directly relating the preintersection numbers to the eigenvalues and multiplicities of a graph. Within this context, in the Appendix of the paper by Van Dam and Haemers [12], the authors wrote the following: "In this appendix we sketch a proof of the following result: for a distance-regular graph the spectrum determines the intersection array. This less-known but relevant result (mentioned in the introduction) has been observed before, but it does not seem to be readily available in the literature."

Their method consists of three steps: first, use the scalar product (2.2) to find the (pre)distance polynomials, as explained in Subsection 3.1 (apply Gram-Schmidt orthogonalisation and the normalization condition); second, compute the distance matrices of the graph by applying the distance polynomials to its adjacency matrix; and third, calculate the intersection parameters from the distance matrices.

However, in our context of a general graph, this method does not apply. The reason is that neither the distance matrices can be obtained from the predistance polynomials, nor the preintersection numbers are related to such matrices. Instead, an alternative would be to compute the predistance polynomials as in Subsection 3.1, and then calculate the preintersection numbers by applying the results of Subsection 3.3. Let us see that, if we follow properly this procedure, we can obtain explicit formulas for the preintersection numbers in terms only of the information given by the spectrum. To this end, we call into play the average numbers of closed walks as a new piece of information. In fact, these averages also determine the spectrum, in the same way as the predistance polynomials and the preintersection numbers do. These averages can be seen as a generalization of the numbers of closed $\ell$-walks in a distance-regular graph, where, for any fixed length $\ell$, they do not depend on the root vertex.

Proposition 3.7. Let $\Gamma$ be a graph. Then, its preintersection numbers can be computed directly from its spectrum $\mathrm{sp} \Gamma$ by using the average number of closed walks of length $\ell$, that is, $c(\ell)=\frac{1}{n} \operatorname{tr}\left(\boldsymbol{A}^{\ell}\right)=\frac{1}{n} \sum_{i=0}^{d} m_{i} \lambda_{i}^{\ell}$, $\ell=0,1,2, \ldots$, and their first values are:

$$
\begin{aligned}
\alpha_{0} & =0, \quad \beta_{0}=\lambda_{0} \\
\gamma_{1} & =\frac{c(2)}{\lambda_{0}}, \quad \alpha_{1}=\frac{c(3)}{c(2)}, \quad \beta_{1}=\lambda_{0}-\alpha_{1}-\gamma_{1}, \\
\gamma_{2} & =\frac{\lambda_{0}\left[c(2) c(4)-c(3)^{2}-c(2)^{3}\right]}{c(2)\left[\lambda_{0}^{2} c(2)-c(3) \lambda_{0}-c(2)^{2}\right]}, \quad \alpha_{2}=\frac{c(2)^{2} c(5)-2 c(2) c(3) c(4)-c(3)^{3}}{c(2)\left[c(2) c(4)-c(3)^{2}-c(2)^{3}\right]}, \quad \beta_{2}=\cdots .
\end{aligned}
$$

Proof. The proof is by induction. We know that, knowing the predistance polynomials $p_{0}, \ldots, p_{i-1}$, $i \geq 1$, the Gram-Schmidt method yields

$$
p_{i}=\frac{r_{i}\left(\lambda_{0}\right)}{\left\|r_{i}\right\|^{2}} r_{i}
$$

where

$$
r_{i}=x^{i}-\sum_{j=0}^{i-1} \frac{\left\langle x^{i}, p_{j}\right\rangle}{\left\|p_{j}\right\|^{2}}=x^{i}-\sum_{j=0}^{i-1} \frac{\sum_{h=0}^{d} m\left(\lambda_{h}\right) \lambda_{h}^{i} p_{j}\left(\lambda_{h}\right)}{\sum_{h=0}^{d} m\left(\lambda_{h}\right) p_{j}^{2}\left(\lambda_{h}\right)}
$$


Then, from $p_{0}=1$, we obtain that $p_{1}=\frac{\lambda_{0}}{c(2)} x$, whence, applying the formulas

$$
\begin{aligned}
\gamma_{i} & =\frac{\left\langle x p_{i-1}, p_{i}\right\rangle}{\left\|p_{i}\right\|^{2}}=\frac{1}{p_{i}\left(\lambda_{0}\right)} \sum_{j=0}^{d} m\left(\lambda_{j}\right) \lambda_{j} p_{i}\left(\lambda_{j}\right) p_{i-1}\left(\lambda_{j}\right), \\
\alpha_{i} & =\frac{\left\langle x p_{i}, p_{i}\right\rangle}{\left\|p_{i}\right\|^{2}}=\frac{1}{p_{i}\left(\lambda_{0}\right)} \sum_{j=0}^{d} m\left(\lambda_{j}\right) \lambda_{j} p_{i}^{2}\left(\lambda_{j}\right), \\
\beta_{i} & =\lambda_{0}-\alpha_{i}-\gamma_{i} .
\end{aligned}
$$

with $i=0,1$ we get (3.16) and (3.17). In general, if all the coefficients of the predistance polynomials $p_{0}, \ldots, p_{i-1}, i \geq 1$, are given in terms of the numbers $c(\ell)$ 's, we proceed in the same way by first calculating $p_{i}$ and then applying the formulas (3.20)-(3.21). This assures that the obtained preintersection parameters $\alpha_{i}, \beta_{i}$, and $\gamma_{i}$ will be expressed also in terms of the $c(\ell)$ 's. For instance, the computation for $i=2$ give the results in (3.18).

4. An example. Let us illustrate the results of Section 3 with one example. Let $\Gamma$ be the graph 4.47 of Table 4 in the textbook of Cvetković, Doob, and Sachs [7], shown in Figure 1, which has $n=9$ vertices, and spectrum

$$
\operatorname{sp} \Gamma=\left\{3^{1},\left(\frac{-1+\sqrt{13}}{2}\right)^{2}, 0^{3},(-1)^{1},\left(\frac{-1-\sqrt{13}}{2}\right)^{2}\right\} .
$$

Thus, $\Gamma$ has $d+1=5$ distinct eigenvalues.

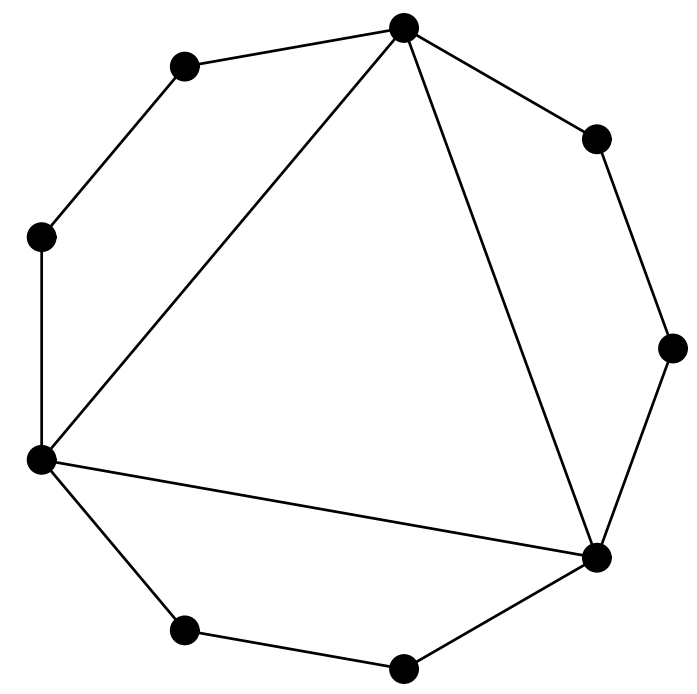

Figure 1. The graph 4.47 in Table 4 of [7]. 
Electronic Journal of Linear Algebra, ISSN 1081-3810

A publication of the International Linear Algebra Society

Volume 36, pp. 629-644, September 2020.

639

Equivalent Characterizations of the Spectra of Graphs and Applications

4.1. From the spectrum to the predistance polynomials. As mentioned in Subsection 3.1, the sequence of predistance polynomials $p_{0}, p_{1}, p_{2}, p_{3}, p_{4}$ are orthogonal with respect to the scalar product

$$
\langle f, g\rangle_{\Gamma}=\frac{1}{n} \operatorname{tr}(f(\boldsymbol{A}) g(\boldsymbol{A}))=\frac{1}{n} \sum_{i=0}^{d} m_{i} f\left(\lambda_{i}\right) g\left(\lambda_{i}\right)
$$

and normalized in such a way that $\left\|p_{i}\right\|_{\Gamma}^{2}=p_{i}\left(\lambda_{0}\right)$. Then, we can obtain them by applying the Gram-Schmidt method, starting from the sequence $1, x, x^{2}, x^{3}, x^{4}$, and the result is:

$$
\begin{aligned}
& p_{0}(x)=1 \\
& p_{1}(x)=\frac{9}{8} x \\
& p_{2}(x)=-\frac{268}{157}-\frac{201}{1256} x+\frac{201}{314} x^{2} \\
& p_{3}(x)=\frac{23607}{50711}-\frac{83082}{50711} x-\frac{732}{2983} x^{2}+\frac{183}{646} x^{3} \\
& p_{4}(x)=\frac{78}{323}+\frac{547}{1292} x-\frac{32}{57} x^{2}-\frac{113}{969} x^{3}+\frac{1}{12} x^{4} .
\end{aligned}
$$

4.2. From the predistance polynomials to the spectrum. To obtain the spectrum from the predistance polynomials, we can use the results in Proposition 3.1. So, the Hoffman polynomial $H=$ $p_{0}+p_{1}+p_{2}+p_{3}+p_{4}$ is

$$
H(x)=-\frac{1}{4} x-\frac{1}{6} x^{2}+\frac{1}{6} x^{3}+\frac{1}{12} x^{4},
$$

with zeros being the distinct eigenvalues different from $\lambda_{0}$ :

$$
\lambda_{1}=\frac{-1+\sqrt{13}}{2}, \quad \lambda_{2}=0, \quad \lambda_{3}=-1, \quad \lambda_{4}=\frac{-1-\sqrt{13}}{2} .
$$

Moreover, the largest root of the polynomial given by $(3.9)$ is $\lambda_{0}=3$. Alternatively, by $(3.6), \lambda_{0}=-\frac{\omega_{1}^{1} \omega_{2}^{0}}{\omega_{2}^{2}}=$ $-(9 / 8)(-268 / 157) /(201 / 314)=3$.

Moreover, the values of the parameters $\phi_{i}$ and $p_{d}\left(\lambda_{i}\right)$ are:

$$
\phi_{0}=108, \quad \phi_{1}=\frac{3}{2}[13-7 \sqrt{13}], \quad \phi_{2}=9, \quad \phi_{3}=-12, \quad \phi_{4}=\frac{3}{2}[13+7 \sqrt{13}],
$$

and

$$
\begin{gathered}
p_{4}\left(\lambda_{0}\right)=\frac{39}{646}, \quad p_{4}\left(\lambda_{1}\right)=-\frac{1}{646}[39+21 \sqrt{13}], \quad p_{4}\left(\lambda_{2}\right)=\frac{78}{323}, \\
p_{4}\left(\lambda_{3}\right)=-\frac{351}{646}, \quad p_{4}\left(\lambda_{4}\right)=\frac{1}{646}[-39+21 \sqrt{13}] .
\end{gathered}
$$

Thus, by applying (3.10), we get the multiplicities:

$$
m_{0}=1, \quad m_{1}=2, \quad m_{2}=3, \quad m_{3}=1, \quad m_{4}=2 .
$$


4.3. From the predistance polynomials to the preintersection numbers. To obtain the preintersection numbers by using the predistance polynomials, we apply Proposition 3.3 giving the relationship between the preintersection matrix and the polynomial coefficient matrix of the graph.

The matrix $\boldsymbol{\Omega}$ containing the polynomial coefficients of the graph $\Gamma$ is:

$$
\boldsymbol{\Omega}=\left(\begin{array}{ccccc}
1 & 0 & 0 & 0 & 0 \\
0 & 9 / 8 & 0 & 0 & 0 \\
-268 / 157 & -201 / 1256 & 201 / 314 & 0 & 0 \\
23607 / 50711 & -83082 / 50711 & -732 / 2983 & 183 / 646 & 0 \\
78 / 323 & 547 / 1292 & -32 / 57 & -113 / 969 & 1 / 12
\end{array}\right)
$$

Then, with $\boldsymbol{U}$ given in (3.11), Proposition 3.3 yields:

$$
\boldsymbol{R}^{\prime}=\boldsymbol{\Omega}^{\prime} \boldsymbol{U} \boldsymbol{\Omega}^{-1}=\left(\begin{array}{ccccc}
0 & 8 / 9 & 0 & 0 & 0 \\
3 & 1 / 4 & 471 / 268 & 0 & 0 \\
0 & 67 / 36 & 387 / 628 & 21641 / 9577 & 0 \\
0 & 0 & 6588 / 10519 & 27036 / 50711 & 1098 / 323
\end{array}\right)
$$

and finally, we add the last row of the matrix $\boldsymbol{R}$ of preintersection numbers by using the equality $\alpha_{i}+\beta_{i}+\gamma_{i}=$ $\beta_{0}=3$ :

$$
\left(\begin{array}{ccccc}
\alpha_{0} & \gamma_{1} & & & \\
\beta_{0} & \alpha_{1} & \gamma_{2} & & \\
& \beta_{1} & \alpha_{2} & \gamma_{3} & \\
& & \beta_{2} & \alpha_{3} & \\
& & & \beta_{3} & \gamma_{4} \\
& & & & \alpha_{4}
\end{array}\right)=\left(\begin{array}{ccccc}
0 & 8 / 9 & & & \\
3 & 1 / 4 & 471 / 268 & & \\
& 67 / 36 & 387 / 628 & 21641 / 9577 & \\
& & 6588 / 10519 & 27036 / 50711 & 1098 / 323 \\
& & & 4082 / 19703 & -129 / 323
\end{array}\right)
$$

4.4. From the preintersection numbers to the predistance polynomials. In order to obtain the predistance polynomials from the preintersection numbers of $\Gamma$, we apply the recurrence (3.12) which, initialized with $p_{0}=1$, yields:

$$
\begin{aligned}
& p_{0}(x)=1, \\
& p_{1}(x)=\frac{\left(x-\alpha_{0}\right) p_{0}-\beta_{(-1)} p_{(-1)}}{\gamma_{1}}=\frac{9}{8} x, \\
& p_{2}(x)=\frac{\left(x-\frac{1}{4}\right) p_{1}-3}{\frac{471}{268}}=-\frac{268}{157}-\frac{201}{1256} x+\frac{201}{314} x^{2}, \\
& p_{3}(x)=\frac{\left(x-\frac{387}{628}\right) p_{2}-\frac{67}{36} p_{1}}{\frac{21641}{9577}}=\frac{23607}{50711}-\frac{83082}{50711} x-\frac{732}{2983} x^{2}+\frac{183}{646} x^{3}, \\
& p_{4}(x)=\frac{\left(x-\frac{27036}{50711}\right) p_{3}-\frac{6588}{10519} p_{2}}{\frac{1098}{323}}=\frac{78}{323}+\frac{547}{1292} x-\frac{32}{57} x^{2}-\frac{113}{969} x^{3}+\frac{1}{12} x^{4} .
\end{aligned}
$$

Alternatively, we can compute the characteristic polynomial of each submatrix $\boldsymbol{R}_{i-1}$ for $i=1, \ldots, d$. 
Electronic Journal of Linear Algebra, ISSN 1081-3810

A publication of the International Linear Algebra Society

Volume 36, pp. 629-644, September 2020.

641

Equivalent Characterizations of the Spectra of Graphs and Applications

Then, we get:

$$
\begin{aligned}
& p_{1}(x)=\frac{9}{8} \operatorname{det}(x \boldsymbol{I}-(0))=\frac{9}{8} x, \\
& p_{2}(x)=\frac{9}{8} \cdot \frac{268}{471} \operatorname{det}\left(x \boldsymbol{I}-\left(\begin{array}{cc}
0 & 8 / 9 \\
3 & 1 / 4
\end{array}\right)\right)=-\frac{268}{157}-\frac{201}{1256} x+\frac{201}{314} x^{2}, \\
& p_{3}(x)=\frac{9}{8} \cdot \frac{268}{471} \cdot \frac{9577}{21641} \operatorname{det}\left(x \boldsymbol{I}-\left(\begin{array}{ccc}
0 & 8 / 9 & \\
3 & 1 / 4 & 471 / 268 \\
& 67 / 36 & 387 / 628
\end{array}\right)\right) \\
& =\frac{23607}{50711}-\frac{83082}{50711} x-\frac{732}{2983} x^{2}+\frac{183}{646} x^{3} \text {, }
\end{aligned}
$$

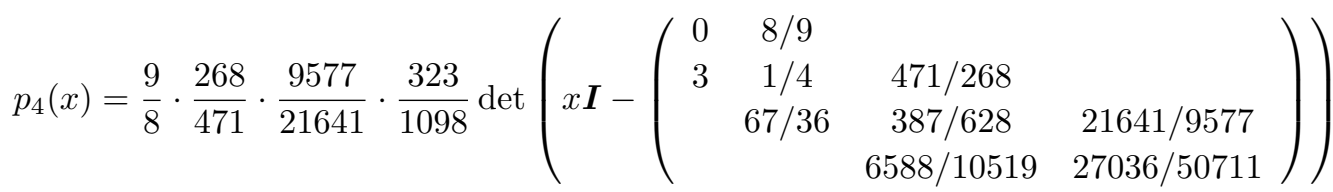

$$
\begin{aligned}
& =\frac{78}{323}+\frac{547}{1292} x-\frac{32}{57} x^{2}-\frac{113}{969} x^{3}+\frac{1}{12} x^{4} \text {. }
\end{aligned}
$$

We can also check that the principal coefficients of each predistance polynomials are easily determined by the parameters $\gamma_{i}$ 's:

$$
\begin{aligned}
& \omega_{1}^{1}=\frac{1}{\gamma_{1}}=\frac{9}{8} \\
& \omega_{2}^{2}=\frac{1}{\gamma_{1} \gamma_{2}}=\frac{9}{8} \cdot \frac{268}{471}=\frac{201}{314} \\
& \omega_{3}^{3}=\frac{1}{\gamma_{1} \gamma_{2} \gamma_{3}}=\frac{9}{8} \cdot \frac{268}{471} \cdot \frac{9577}{21641}=\frac{183}{646} \\
& \omega_{4}^{4}=\frac{1}{\gamma_{1} \gamma_{2} \gamma_{3} \gamma_{4}}=\frac{9}{8} \cdot \frac{268}{471} \cdot \frac{9577}{21641} \cdot \frac{323}{1098}=\frac{1}{12}
\end{aligned}
$$

4.5. From the preintersection numbers to the spectrum. To obtain the spectrum of $\Gamma$, we first compute the characteristic polynomial of the preintersection matrix

$$
\boldsymbol{R}=\left(\begin{array}{ccccc}
0 & 8 / 9 & & & \\
3 & 1 / 4 & 471 / 268 & & \\
& 67 / 36 & 387 / 628 & 21641 / 9577 & \\
& & 6588 / 10519 & 27036 / 50711 & 1098 / 323 \\
& & & 4082 / 19703 & -129 / 323
\end{array}\right)
$$

which turns out to be $\phi_{\Gamma}(x)=x^{5}-x^{4}-8 x^{3}+3 x^{2}+9 x$. Then, its roots are

$$
\lambda_{0}=3, \quad \lambda_{1}=\frac{1}{2}(-1+\sqrt{13}), \quad \lambda_{2}=0, \quad \lambda_{3}=-1, \quad \lambda_{4}=\frac{1}{2}(-1-\sqrt{13}) .
$$

To compute the multiplicities, we first consider the left and right eigenvectors of $\lambda_{0}$ :

$$
\boldsymbol{u}_{0}=\boldsymbol{j}=(1,1,1,1,1) \quad \text { and } \quad \boldsymbol{v}_{0}=\left(1, \frac{27}{8}, \frac{4489}{1256}, \frac{100467}{101422}, \frac{39}{646}\right)^{\top},
$$


so giving $n=\left\langle\boldsymbol{u}_{0}, \boldsymbol{v}_{0}\right\rangle=9$. Now, let us consider, for example, the eigenvalue $\lambda_{2}=0$. Then, the corresponding left and right normalized eigenvectors of $\boldsymbol{R}$ are

$$
\boldsymbol{u}_{2}=\left(1,0,-\frac{32}{67}, 0, \frac{86}{183}, 4\right) \quad \text { and } \quad \boldsymbol{v}_{2}=\left(1,0,-\frac{286}{157}, \frac{23607}{50711}, \frac{78}{323}\right)^{\top} .
$$

Then, we get

$$
m_{2}=\frac{n}{\left\langle\boldsymbol{u}_{2}, \boldsymbol{v}_{2}\right\rangle}=3
$$

and similar computations give $\left\langle\boldsymbol{u}_{1}, \boldsymbol{v}_{1}\right\rangle=\frac{9}{2},\left\langle\boldsymbol{u}_{3}, \boldsymbol{v}_{3}\right\rangle=9$, and $\left\langle\boldsymbol{u}_{4}, \boldsymbol{v}_{4}\right\rangle=\frac{9}{2}$, so giving the other multiplicities $m_{1}=2, m_{3}=1$, and $m_{4}=2$.

4.6. From the spectrum to the preintersection numbers. In our case, the average numbers of walks of length $\ell=0,1, \ldots, 5$ turn out to be

$$
c(0)=1, \quad c(1)=0, \quad c(2)=\frac{8}{3}, \quad c(3)=\frac{2}{3}, \quad c(4)=16, \quad c(5)=\frac{40}{3},
$$

and, then, Proposition 3.7 gives:

$$
\alpha_{0}=0, \quad \beta_{0}=3, \quad \gamma_{1}=\frac{8}{9}, \quad \alpha_{1}=\frac{1}{4}, \quad \beta_{1}=\frac{67}{36}, \quad \gamma_{2}=\frac{471}{268}, \quad \alpha_{2}=\frac{387}{628}, \quad \beta_{2}=\frac{6588}{10519}, \ldots
$$

and we can keep applying the method to obtain the remaining preintersection numbers.

5. Some applications. In this section, we present some applications of the information given by the spectrum, the predistance polynomials, and the preintersection numbers of a given graph. Moreover, we show how the equivalences of these pieces of information allows us to rewrite some of the properties or conditions in different forms. We begin with some combinatorial properties of a graph that can be deduced from its preintersection numbers (see [1]).

5.1. Properties of the preintersection numbers. We can see if the graph is bipartite, or how large is its odd girth, with simply checking at its matrix $\Omega$ of coefficients.

Proposition 5.1. Let $\Gamma$ be a graph with $d+1$ distinct eigenvalues. Then,

(a) $\Gamma$ is bipartite if and only if $\alpha_{0}=\cdots=\alpha_{d}=0$.

(b) If $\Gamma$ is not bipartite, then it has odd girth $2 m+1$ if and only if $\alpha_{0}=\cdots=\alpha_{m-1}=0$ and $\alpha_{m}>0$.

From the results in Subsection 3.3, we observe that if $\alpha_{i}=0$ for $0 \leq i \leq m$ then the coefficients of the preintersection polynomials $\omega_{i}^{j}$ equals 0 if $i+j$ is odd (that is, when $i$ and $j$ have distinct parity). Then, we can rewrite the previous proposition as follows:

\section{Proposition 5.2.}

(a) A graph $\Gamma$ with $d+1$ distinct eigenvalues is bipartite if and only if, in the matrix $\Omega, \omega_{i}^{j}=0$ for every $i+j$ odd.

(b) If $\Gamma$ is not bipartite, then it has odd girth $2 m+1$ if and only if $\omega_{i}^{j}=0$ for every $i+j$ odd and $i \leq m$.

Concerning the girth, we have the following results. 
Electronic Journal of Linear Algebra, ISSN 1081-3810

A publication of the International Linear Algebra Society

Volume 36, pp. 629-644, September 2020.

Proposition 5.3.

(a) A regular graph $\Gamma$ has girth $2 m+1$ if and only if $\alpha_{0}=\cdots=\alpha_{m-1}=0, \alpha_{m} \neq 0$ and $\gamma_{1}=\cdots=$ $\gamma_{m}=1$.

(b) $A$ regular graph $\Gamma$ has girth $2 m$ if and only if $\alpha_{0}=\cdots=\alpha_{m-1}=0, \gamma_{1}=\cdots=\gamma_{m-1}=1$ and $\gamma_{m}>1$.

Using again the results of Subsection 3.3, we have:

Proposition 5.4.

(a) A regular graph $\Gamma$ has girth $2 m+1$ if and only if the polynomial coefficients satisfies $\omega_{i}^{j}=0$ for every $i+j$ odd and $i \leq m-1, \omega_{m}^{j} \neq 0$ for some $m+j$ odd, and $\omega_{1}^{1}=\cdots=\omega_{m}^{m}=1$.

(b) A regular graph $\Gamma$ has girth $2 m$ if and only if the polynomial coefficients satisfies $\omega_{i}^{j}=0$ for every $i+j$ odd and $i \leq m-1, \omega_{1}^{1}=\cdots=\omega_{m-1}^{m-1}=1$, and $\omega_{m}^{m}<1$.

5.2. Characterizations of distance-regularity. Now we give some characterizations of distanceregularity in graphs, which are given in terms of the different pieces of information considered. We begin with the so-called 'spectral excess theorem' (see Fiol and Garriga [19]), which can be seen as a quasi-spectral characterization of a distance-regular graph.

THEOREM 5.5. (The spectral excess theorem) A regular graph $\Gamma=(V, E)$ is distance-regular if an only if its spectral excess

$$
p_{d}\left(\lambda_{0}\right)=\frac{\beta_{0} \beta_{1} \cdots \beta_{d-1}}{\gamma_{1} \gamma_{2} \cdots \gamma_{d}}=n\left(\sum_{i=0}^{d} \frac{\pi_{0}^{2}}{m_{i} \pi_{i}^{2}}\right)^{-1}
$$

(where $\pi_{i}=\prod_{j \neq i}\left|\lambda_{i}-\lambda_{j}\right|$, for $\left.i=0, \ldots, d\right)$ equals the average excess

$$
\bar{k}_{d}=\frac{1}{n} \sum_{u \in V}\left|\Gamma_{d}(u)\right|
$$

Proof. The result was proved in [19] with the spectral excess $p_{d}\left(\lambda_{0}\right)$ given in terms of the spectrum. The condition involving the preintersection numbers comes from applying Lemma 2.1 (a2) starting from $p_{1}\left(\lambda_{0}\right)=1$.

The following result was proved by Abiad, Van Dam, and Fiol [1] for a more particular family of distanceregular graphs. (Here it can be shown that the conditions on the preintersection numbers $\gamma_{i}$ 's is related to the existence of unique geodetic paths between vertices.)

THEOREM 5.6. Let $\Gamma$ be a graph with $d+1$ distinct eigenvalues and preintersection numbers $\gamma_{i}$, $i=$ $1, \ldots, d$.

(a) If $\gamma_{1}=\cdots=\gamma_{d-1}=1$, then $\Gamma$ is distance-regular.

(b) If $\Gamma$ is bipartite and $\gamma_{1}=\cdots=\gamma_{d-2}=1$, then $\Gamma$ is distance-regular.

This result implies that a graph is distance-regular if its predistance polynomials are monic.

THEOREM 5.7. Let $\Gamma$ be a graph with $d+1$ distinct eigenvalues and predistance polynomials $p_{i}$, $i=$ $0,1, \ldots, d$. 
(a) If all the $p_{i}$ 's, are monic $\left(\omega_{i}^{i}=1\right)$ for $i=1, \ldots, d-1$, then $\Gamma$ is distance-regular.

(b) If $\Gamma$ is bipartite and all the $p_{i}$ 's, are monic, then $\Gamma$ is distance-regular.

Proof. Apply Theorem 5.6 and Proposition 3.2 (c) recursively from $\omega_{0}^{0}=1$.

\section{REFERENCES}

[1] A. Abiad, E.R. van Dam, and M.A. Fiol. Some spectral and quasi-spectral characterizations of distance-regular graphs. $J$. Combin. Theory Ser. A, 143:1-18, 2016.

[2] N. Biggs. Algebraic Graph Theory. Cambridge University Press, Cambridge, 1974; second edition, 1993.

[3] A.E. Brouwer, A.M. Cohen, and A. Neumaier. Distance-Regular Graphs. Springer-Verlag, Berlin, 1989.

[4] A. E. Brouwer and W.H. Haemers. The Gewirtz graph: An exercise in the theory of graph spectra. European J. Combin., 14:397-407, 1993

[5] A.E. Brouwer and W.H. Haemers. Spectra of Graphs. Springer, 2012. Available at http://homepages.cwi.nl/ aeb/math/ $\mathrm{ipm} /$.

[6] M. Cámara, J. Fàbrega, M.A. Fiol, and E. Garriga. Some families of orthogonal polynomials of a discrete variable and their applications to graphs and codes. Electron. J. Combin., 16(1):Article R83, 2009.

[7] D.M. Cvetković, M. Doob, and H. Sachs. Spectra of Graphs. Theory and Application, second edition. VEB Deutscher Verlag der Wissenschaften, Berlin, 1982.

[8] C. Dalfó, E.R. van Dam, M.A. Fiol, E. Garriga, and B.L. Gorissen. On almost distance-regular graphs. J. Combin. Theory Ser. A, 118(3):1094-1113, 2011

[9] C. Dalfó, M.A. Fiol, and E. Garriga. Characterizing $(\ell, m)$-walk-regular graphs. Linear Algebra Appl., 433(9):1821-1826, 2010.

[10] E.R. van Dam. The spectral excess theorem for distance-regular graphs: A global (over)view. Electron. J. Combin., 15(1):Article R129, 2008.

[11] E.R. van Dam and M.A. Fiol. A short proof of the odd-girth theorem, Electron. J. Combin., 19(3):Article P12, 2012.

[12] E.R. van Dam and W.H. Haemers. Spectral characterizations of some distance-regular graphs. J. Algebraic Combin., 15:189-202, 2002.

[13] E.R. van Dam and W.H. Haemers. An odd characterization of the generalized Odd graphs. J. Combin. Theory Ser. B, 101:486-489, 2011.

[14] E.R. van Dam and J.H. Koolen. A new family of distance-regular graphs with unbounded diameter. Invent. Math., 162:189193, 2005.

[15] E.R. van Dam, J.H. Koolen, and H. Tanaka. Distance-regular graphs. Electron. J. Combin., Article DS22, 2016.

[16] M.A. Fiol. An eigenvalue characterization of antipodal distance-regular graphs. Electron. J. Combin., 4(1):Article R30, 1997.

[17] M.A. Fiol. Algebraic characterizations of distance-regular graphs. Discrete Math., 246:111-129, 2002.

[18] M.A. Fiol, S. Gago, and E. Garriga. A simple proof of the spectral excess theorem for distance-regular graphs. Linear Algebra Appl., 432(9):2418-2422, 2010.

[19] M.A. Fiol and E. Garriga. From local adjacency polynomials to locally pseudo-distance-regular graphs. J. Combin. Theory Ser. B, 71:162-183, 1997.

[20] C.D. Godsil. Algebraic Combinatorics. Chapman and Hall, New York, 1993.

[21] A.J. Hoffman. On the polynomial of a graph. Amer. Math. Monthly, 70:30-36, 1963.

[22] H. Kurihara and H. Nozaki. A spectral equivalent condition of the P-polynomial property for association schemes. Electron. J. Combin., 21(3):Article P3.1, 2014.

[23] G.-S. Lee and C.-W. Weng. The spectral excess theorem for general graphs. J. Combin. Theory Ser. A, 119:1427-1431, 2012.

[24] P. Rowlinson. Linear algebra. In: L.W. Beineke and R.J. Wilson (editors), Graph Connections, Oxford Lecture Ser. Math. Appl., Vol. 5, 86-99, Oxford University Press, New York, 1997. 